\title{
Development of a Multiplexed Interface for Capillary Electrophoresis-Electrospray Ion Trap Mass Spectrometry
}

\author{
Fu-An Li, Ming-Chi Wu, and Guor-Rong Her* \\ Department of Chemistry, National Taiwan University, Taipei, Taiwan, R.O.C
}

\begin{abstract}
A four-channel multiplexed electrospray capillary electrophoresis interface has been developed. This new interface permits up to four capillary electrophoresis columns to be sampled sequentially by means of a stepper motor and a notched rotating plate assembly, which at any instant occludes all but a single sprayer. In this design, four sheath liquid electrospray probes are oriented in a circular array situated $90^{\circ}$ relative to one another. The rotating metal disk, which contains a one-quarter notch, is mounted to the stepper motor assembly and is located between the sprayers and the entrance aperture of an ion trap mass spectrometer. By using the data acquisition signal from the ion trap mass spectrometer, the scan event is synchronized with the rotation of the metal disk. With this device, four discrete sample streams can be simultaneously analyzed, resulting in a 4-fold increase in analytical throughput.
\end{abstract}

Within the current scope of drug discovery and development, applications requiring rapid identification and characterization of large numbers of compounds are increasing. Examples include chemical synthesis screening, DNA characterization, and proteomics. As a result, there is a continued need for high-throughput qualitative and quantitative analysis. Because of the demand for fast analysis, high separation efficiency (in the range of hundreds of thousands of plates), small sample volumes (in the nanoliter range), and high sensitivity, capillary electrophoresis (CE) has become an important tool for the analysis of a wide variety of biological and biomedical substances. Unfortunately, despite the high separation efficiency of $\mathrm{CE}$, the throughput is limited to one sample at a time. In recent years, the demand for increased throughput has prompted the development of high-throughput $\mathrm{CE}$ methods. ${ }^{1-5}$ Noteworthy among theses approaches is capillary array electrophoresis $(\mathrm{CAE})$, which has proven to be quite

* Corresponding author. Phone: (886) 2-33661647. Fax: (886) 2-23638058. E-Mail: grher@ntu.edu.tw.

(1) Kelly, R. T.; Woolley, A. T. Anal. Chem. 2005, 77, 96A-102A.

(2) Carrilho, E. Electrophoresis 2000, 21, 55-65.

(3) Scherer, J. R.; Kheterpal, I.; Radhakrishnan, A.; Ja, W. W.; Mathies, R. A. Electrophoresis 1999, 20, 1508-1517.

(4) Emrich, C. A.; Tian, H.; Medintz, I. L.; Mathies, R. A. Anal. Chem. 2002, 74, 5076-5083.

(5) Preisler, J.; Hu, P.; Rejtar, T.; Moskovets, E.; Karger, B. L. Anal. Chem. 2002, 74, 17-25. successful in the analysis of a variety of samples. ${ }^{6-8}$ This particular example, which involves simultaneous analysis by several capillaries, illustrates the importance of parallel analysis to maximize the utility of $\mathrm{CE}$.

Because of its sensitivity, capability of sample identification, structure elucidation, and quantification, mass spectrometry (MS) has become an indispensable tool for biomedical and biological research; however, in published applications utilizing CAE systems, analyte detection has involved only optical detectors. Among the MS ionization techniques, electrospray ionization (ESI) is by far the most frequently used technique, primarily because it allows molecules in the liquid phase to be transferred directly into ions in the gas phase. The coupling of ESI-MS with liquid based separation techniques offers high-resolution analysis of complex mixtures (time and mass separation) and information-rich detection..$^{9-11}$ In the past decade, several interfaces have been developed for coupling CE to ESI-MS; however, coupling multiplexed CE with ESI-MS has not been demonstrated.

To increase the throughput of chromatographic methods, parallel separation techniques have become popular. Under parallel chromatography, two or more columns are run simultaneously, with detection by a single mass spectrometer. ${ }^{12-15}$ Two general methods have been reported to accommodate multiple flows into a common mass spectrometer interface: (1) serial highperformance liquid chromatography (HPLC) with a single sprayer ${ }^{14}$ or (2) parallel HPLC with multiple sprayers..$^{15}$ The former approach involves multiple injections staggered in time onto separate columns such that the chromatographic window of interest is always eluting from one of the columns. The effluent from a particular column having the analytes of interest eluting is diverted to a common ESI sprayer by a valve selector. In this mode, a valve selector is used to consecutively monitor a finite chromatographic

(6) Crabtree, H. J.; Bay, S. J.; Lewis, D. F.; Coulson, L. D.; Fitzpatrick, G.; Harrison, D. J.; Delinger, S. L.; Zhang, J. Z.; Dovichi, N. J. Electrophoresis 2000, 21, 1329-1335.

(7) Kang, S. H.; Gong, X.; Yeung, E. S. Anal. Chem. 2000, 72, 3014-3021.

(8) Ma, L.; Gong, X.; Yeung, E. S. Anal. Chem. 2000, 72, 3383-3387.

(9) Moini, M. Anal. Bioanal. Chem. 2002, 373, 466-480.

(10) Brocke, A. V.; Nicholson, G.; Bayer, E. Electrophoresis 2001, 22, 12511266.

(11) Gelpi, E. J. Mass Spectrom. 2002, 37, 241-253.

(12) Hopfgartner, G.; Bourgogne, E. Mass Spectrom. Rev. 2003, 22, 195-214.

(13) Zeng, L.; Kassel, D. B. Anal. Chem. 1998, 70, 4380-4388.

(14) Van Pelt, C. K.; Corso, T. N.; Schultz, G. A.; Lowes, S.; Henion, J. Anal. Chem. 2001, 73, 582-588.

(15) Wang, T.; Zeng, L.; Cohen, J.; Kassel, D. B. Comb. Chem. High Throughput Screening 1999, 2, 327-334.

10.1021/ac052272w CCC: $\$ 33.50$ (c) 2006 American Chemical Society Published on Web 07/04/2006 


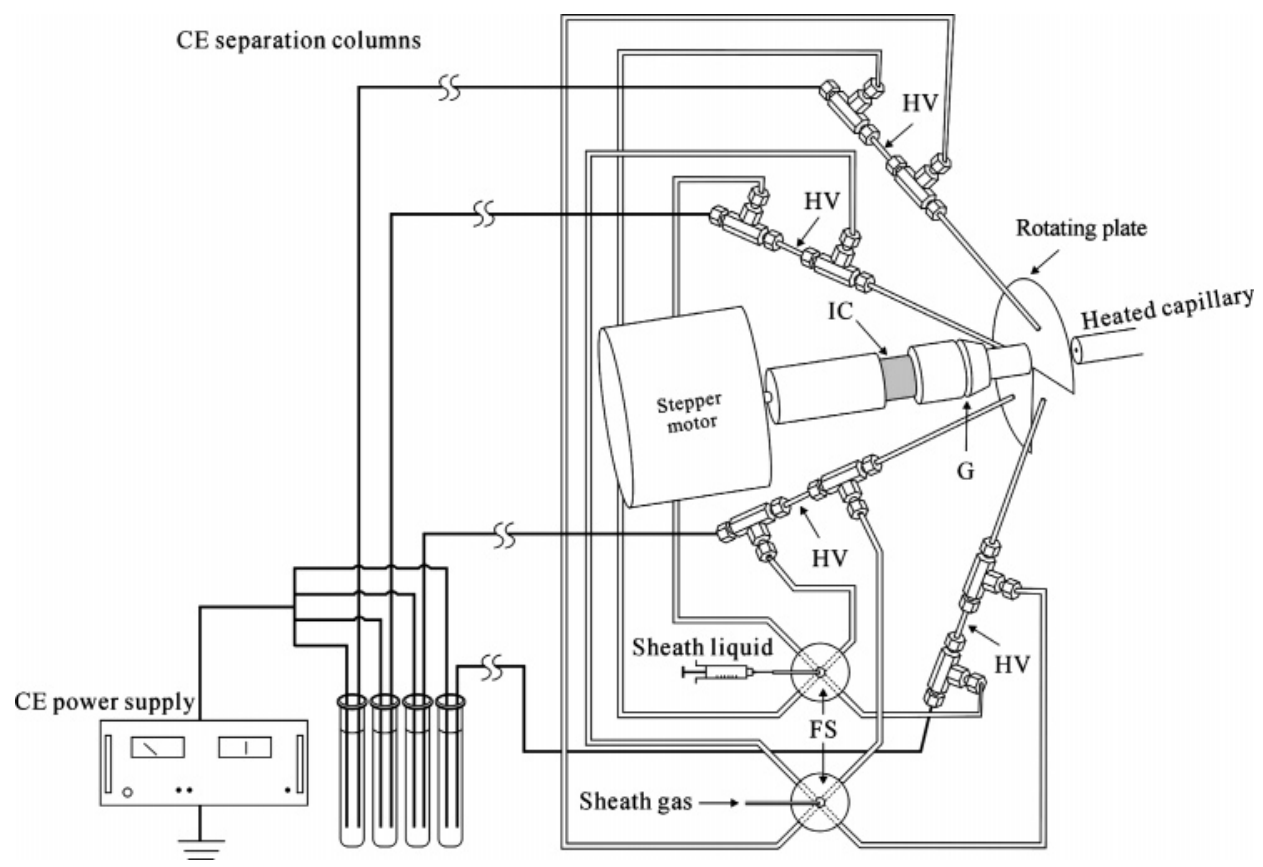

Figure 1. Schematic representation of the multi-CE/ESI-MS interface. HV, ESI high voltage contact; IC, insulating connector; FS, flow splitter; $\mathrm{G}$, grounded brush contact.

window of interest taking advantage of the dead time in the chromatogram. In the second approach, each parallel column has a dedicated ESI sprayer. Parallel chromatography occurs with simultaneous injection, and a mechanical device is used to block all sprayers but one such that only a single sprayer is sampled at any particular instant. Under this design, it is possible to acquire a full chromatogram for each effluent stream, although the sampling frequency for any single column is decreased by a factor of 4 .

One of the main disadvantages of the single sprayer mode is the system carryover. This is one of the advantages of a multisprayer interface, such as the MUX interface system commercialized by Micromass, Inc., although it is important to note that a finite level of crosstalk between sprayers does occur. ${ }^{16,17}$ This system enables the coupling of either four or eight HPLC columns in parallel with an ESI mass spectrometer. Results on the use of the MUX interface have been published, ${ }^{18-21}$ especially in the area of combinatorial chemistry.

In this paper, we present a multiple sheath-flow inlet interface for coupling four CE columns with an ion trap mass spectrometer. This interface permits up to four capillary electrophoresis columns to be sampled sequentially by means of a stepper motor and a notched rotating plate assembly. By using the signal from a printed circuit board (PCB) of the ion trap mass spectrometer as the trigger, synchronization of data acquisition and plate rotation was

(16) Biasi, V. D.; Haskins, N.; Organ, A.; Bateman, R.; Giles, K.; Jarvis, S. Rapid Commun. Mass Spectrom. 1999, 13, 1165-1168.

(17) Bayliss, M. K.; Little, D.; Mallett, D. N.; Plumb, R. S. Rapid Commun. Mass Spectrom. 2000, 14, 2039-2045.

(18) Feng, B.; McQueney, M. S.; Mezzasalma, T. M.; Slemmon, J. R. Anal. Chem. 2001, 73, 5691-5697.

(19) Fang, L.; Demee, M.; Cournoyer, J.; Sierra, T.; Young, C.; Yan, B. Rapid Commun. Mass Spectrom. 2003, 17, 1425-1432.

(20) Xu, R.; Wang, T.; Isbell, J.; Cai, Z.; Sykes, C.; Brailsford, A.; Kassel, D. B. Anal. Chem. 2002, 74, 3055-3062.

(21) Xu, R.; Nemes, C.; Jenkins, K. M.; Rourick, R. A.; Kassel, D. B.; Liu, C. Z. C. J. Am. Soc. Mass Spectrom. 2002, 13, 155-165. achieved. Data from a multiplexed electropherogram were recorded using the "Event Scan" function of the data acquisition software of the ion trap mass spectrometer. Preliminary results reveal that this device enables simultaneous analysis of up to four discrete samples, with a concomitant 4-fold increase in analytical throughput. The potential and limitations of this multiplexed CE/ MS interface are discussed.

\section{EXPERIMENTAL SECTION}

Chemical Reagents. All chemical standards, 4-acetaminophenol $(\mathrm{MW}=151)$, ketoprofen $(\mathrm{MW}=254)$, mefenamic acid (MW $=241)$, niflumic acid ( $\mathrm{MW}=282$ ), oxyphenbutazone ( $\mathrm{MW}=324$ ), phenylbutazone $(\mathrm{MW}=308)$, prednisolone $(\mathrm{MW}=360)$, and sulindac (MW = 356) were obtained from the Laboratories of Foods and Drugs, Department of Health, Taipei, Taiwan. Ammonium acetate, formic acid, sodium hydroxide, and methanol (HPLC grade) were purchased from J. T. Baker (Phillipsburg, $\mathrm{NJ}$ ) and used without further purification. Deionized water (Milli-Q water system; Millipore Inc., Bedford, MA) was used in the preparation of the buffer solution and sheath liquid. All chemical standards were dissolved in methanol, and the concentration of each sample was $250 \mathrm{ppm}$ for multiplexed CE/MS analysis.

Multiplexed CE/MS Interface. The four-channel multiplexed ESI capillary electrophoresis interface (Figure 1) consisted of four sheath-flow sprayers and a rotating plate assembly. The four homemade sheath-flow $\mathrm{CE} / \mathrm{MS}$ sprayers and the rotating plate assembly were mounted on an aluminum stand $(40 \mathrm{~cm} \times 40 \mathrm{~cm}$ $\times 2 \mathrm{~cm})$. Each sprayer consisted of one separation column $(80$ $\mathrm{cm} \times 50-\mu \mathrm{m}$ i.d. $\times 375-\mu \mathrm{m}$ o.d. fused-silica capillary, Polymicro Technologies, Phoenix, AZ) and two stainless steel tubes, which were assembled by two stainless steel "tees" (ss-100-3; Swagelok, Solon, $\mathrm{OH})$. The inner stainless steel tube (0.017-in. i.d. $\times 0.025$ in. o.d. $\times 12.5$ cm; Small Parts Inc., Miami Lakes, FL) was used for delivering sheath liquid, and the outer stainless steel tube (0.032-in. i.d. $\times$ 0.042-in. o.d. $\times 7.5 \mathrm{~cm}$, Small Parts Inc.) was used 


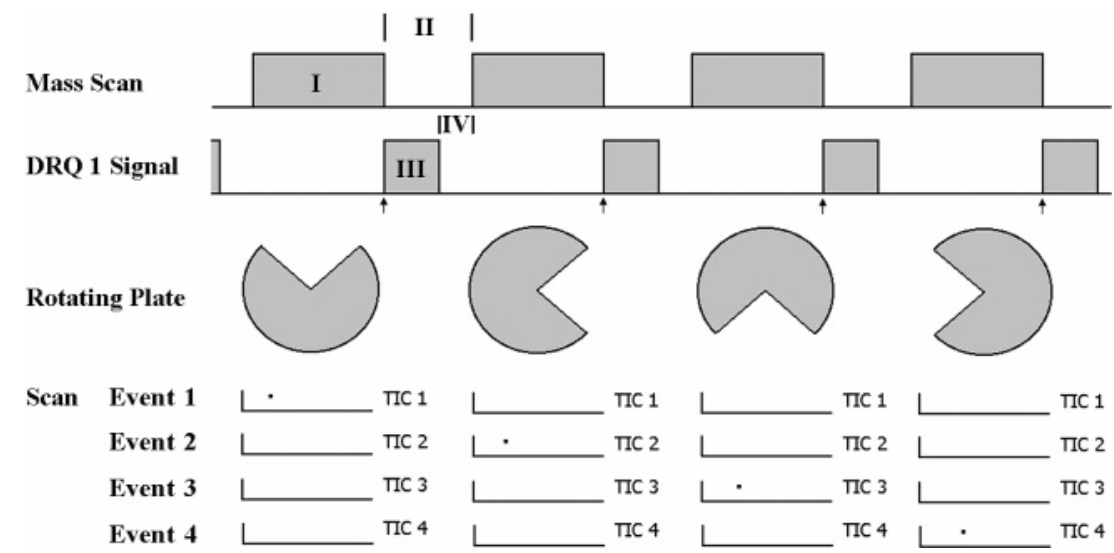

Figure 2. The synchronization between disk motion and scan event. The arrow indicates the trigger for the disk moving to the next position. I, scan time; II, lag time; III, data-uploading time; IV, interscan delay time. The dot represents a MS scan from a particular column.

\section{(a) Column 1}

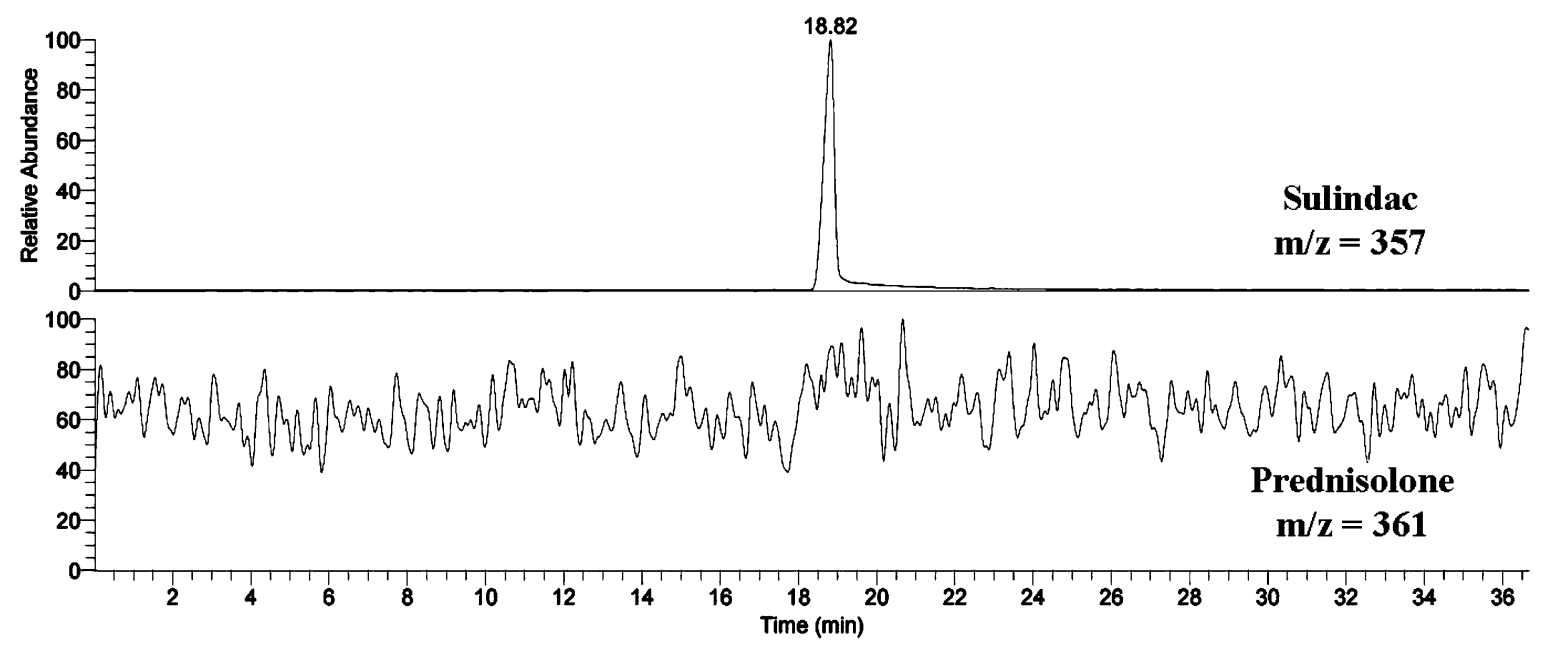

(b) Column 2

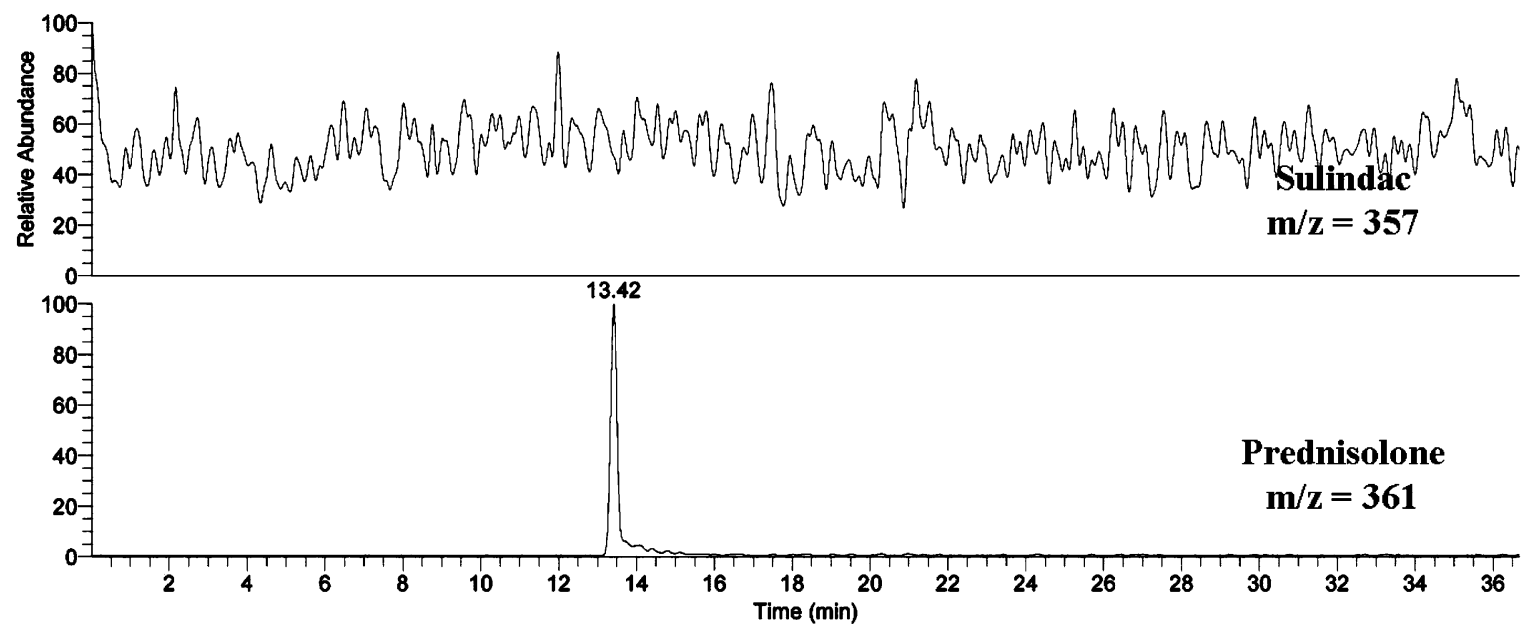

Figure 3. Multi-CE/ESI-MS analysis of two separation columns. (a) Extracted ion electropherogram $(\mathrm{m} / \mathrm{z}=357$ and 361$)$ recorded within event 1 and $(b)$ extracted ion electropherogram $(\mathrm{m} / \mathrm{z}=357$ and 361$)$ recorded within event 2. Sample: column 1, $250 \mathrm{ppm}$ sulindac $\left([\mathrm{M}+\mathrm{H}]^{+}\right.$ $=357)$; column 2, $250 \mathrm{ppm}$ prednisolone $\left([\mathrm{M}+\mathrm{H}]^{+}=361\right)$. Conditions: running buffer composition, $40 \mathrm{mM}$ ammonium acetate buffer at pH 9; sheath liquid, $\mathrm{H}_{2} \mathrm{O} / \mathrm{MeOH} / \mathrm{HCOOH}(80 / 20 / 0.1, \mathrm{v} / \mathrm{v} / \mathrm{v})$ at a flow rate of $5 \mu \mathrm{L} / \mathrm{min}$; sheath gas, $\mathrm{N}_{2}$ at a flow rate of 20 (arbitrary units).

for nebulization gas. The sheath liquid and the nebulization gas were split evenly to the four sprayers by a flow splitter (Z4M1; Valco Instruments Co. Inc., Houston, TX). The four sheath-flow sprayers were oriented in a circular array, in which each sprayer was situated $90^{\circ}$ relative to one another. The sprayers were positioned at a distance of $\sim 15 \mathrm{~mm}$ from the mass spectrometer 
(a)
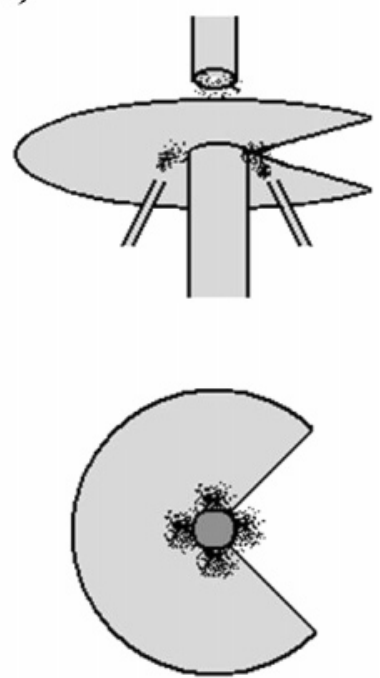

(b)
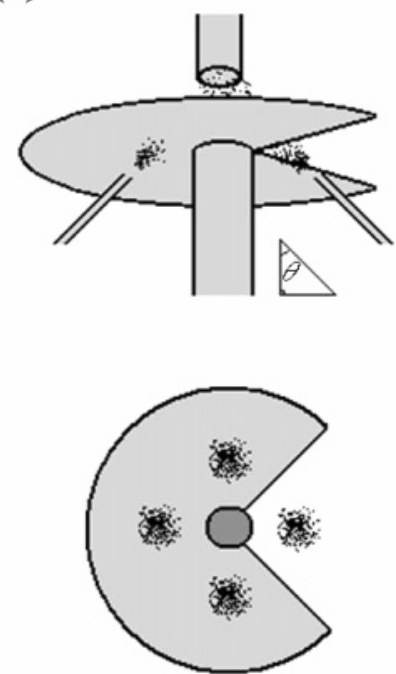

(c)
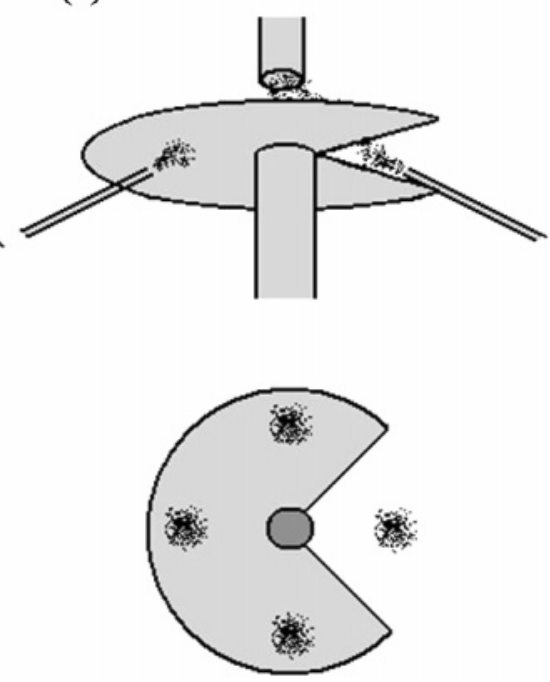

Figure 4. Schematics of the three different sprayer arrangements.

entrance aperture. A metal disk with a $90^{\circ}$ open (notched) section was inserted between the sprayers and the mass spectrometer entrance aperture and was mounted on a stepper motor (TECO Corp., Taiwan). This metal disk also served as the counter electrode of ESI high voltage through a brush connection to ground. The stepper motor was controlled by a personal computer through LabVIEW software (National Instrument Corp., Austin, TX) and a motion control card (National Instrument Corp.) equipped with four analogue input channels. The disk was programmed to rotate in either $180^{\circ}$ or $90^{\circ}$ steps depending on whether two or four sprayers were used.

The rotation of the metal disk was triggered by the DRQ1 (Data ReQuest 1) signal from one of the PCBs in the ion trap mass spectrometer. The DRQ1 signal was fed to the analogue input of the motion control card and was used by the LabVIEW program to synchronize the scan event and the rotation of the stepper motor. The rotation of the disk was controlled by the LabVIEW program so that the open section was rotated to the next sprayer just prior to the start of a new scan. In the starting position, the open section of the disk was oriented in front of sprayer no. 1, and data acquisition was initiated at this location for a period of a single scan. Following the first scan, the disk was rotated $90^{\circ}$ to a new position to allow the mass spectrometer to sample the next sprayer. This process was repeated throughout the course of a $\mathrm{CE}$ analysis to permit the four sprayers to be sampled sequentially and to allow four complete elctropherograms to be simultaneously recorded and independently tracked by the data system.

Multiplexed CE/MS Analysis. An ion trap mass spectrometer (LCQ, Finnigan MAT) operated in the positive ion mode was used throughout this study. MS data were recorded over a massto-charge range of $300 \mathrm{amu}$ using three microscans under automatic gain control (AGC). For data acquisition, the Event Scan function acquired mass spectra from discrete probes as individual scan events within a single acquisition segment. In this manner, the data from four sprayers could be recorded in one file at the same segment of time without modifying the LCQ software.

In the four-column mode of operation, the sheath liquid and sheath gas were split evenly between the sprayers utilizing a four- way stainless steel flow splitter. The flow rates of sheath liquid and sheath gas were set at $5 \mu \mathrm{L} / \mathrm{min}$ and 20 (arbitrary units), respectively. The separation buffer was $40 \mathrm{mM}$ ammonium acetate in aqueous solution. The $\mathrm{pH}$ of the solution was adjusted to 9.0 using $\mathrm{NH}_{4} \mathrm{OH}$. Between runs, the capillary was flushed with 500 $\mu \mathrm{L}$ of separation buffer using a syringe pump and then equilibrated for 15 min. The sheath liquid $\mathrm{MeOH} / \mathrm{H}_{2} \mathrm{O} / \mathrm{HCOOH}(80 / 20 / 0.1$, $\mathrm{v} / \mathrm{v} / \mathrm{v}$ ) was delivered by a syringe pump. The four sample solutions were simultaneously injected by raising the sample reservoir $10 \mathrm{~cm}$ for a duration of $15 \mathrm{~s}$. CE/ESI-MS analyses were achieved by applying $+24 \mathrm{kV}$ (high voltage power supply, CZE1000R; Spellman, Valhalla, NY) and $+4 \mathrm{kV}$ to the injection end of the column and the sprayer, respectively.

Safety Considerations. The $\mathrm{CE}$ high voltage should be used with caution.

\section{RESULTS AND DISCUSSION}

A key problem encountered in the construction of the multiplexed CE/MS interface was synchronization of the scan event with the rotation of the metal disk. Unlike a quadrupole mass spectrometer, which has a fixed time for each scan, the scan time of the LCQ ion trap mass spectrometer varies according to the signal present and the setting used for AGC. Under AGC, a prescan is employed to gauge the intensity of the signal entering the trap, and the injection time is inversely varied with the signal to avoid saturation and defocusing that results from space-charge effects between like-charged ions. This feature is vitally important to chromatographic data acquisition, since the analyte concentration can differ dramatically during peak elution.

To synchronize the scan and the rotation of the disk, the signals from the ion trap mass spectrometer were studied. The result suggested that the signal of DRQ1 from one of the PCBs could be used to synchronize the scan event with the stepper motor. Figure 2 shows an illustration of how the rotating plate moves according to the DRQ1 signal. In the mass scan trace, the high-level signal (I) represents the scan time, and the low-level signal (II) represents the time for data-uploading (III) and interscan delay time (IV). As shown in Figure 2, the DRQ1 signal (from one of the test point of the data acquisition DSP board) 

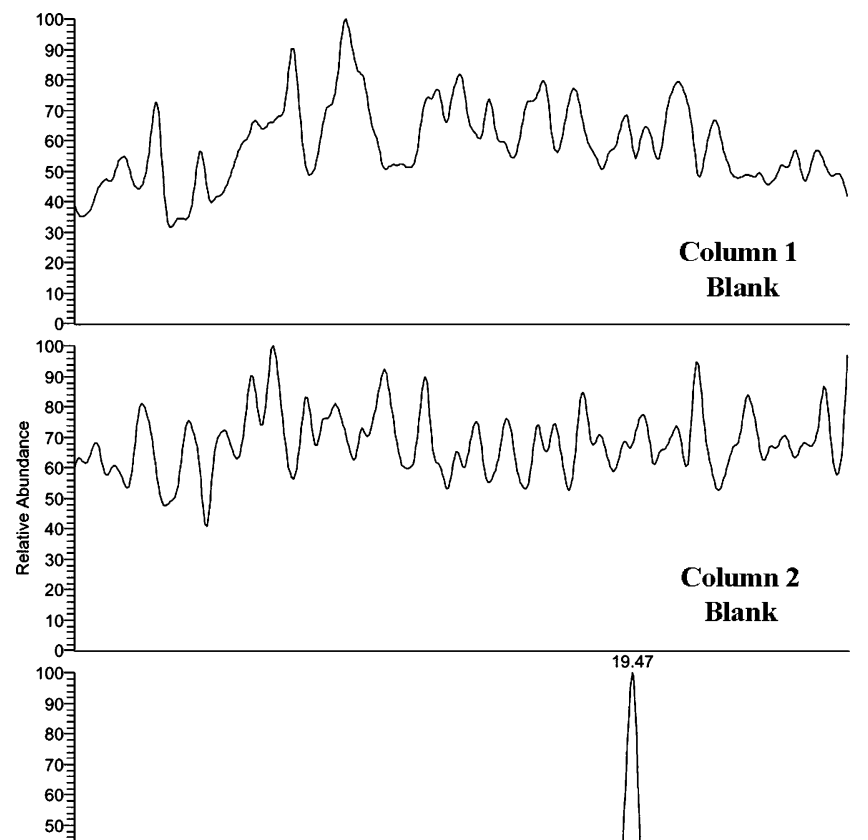

Column 3

2000ppm Niflumic acid

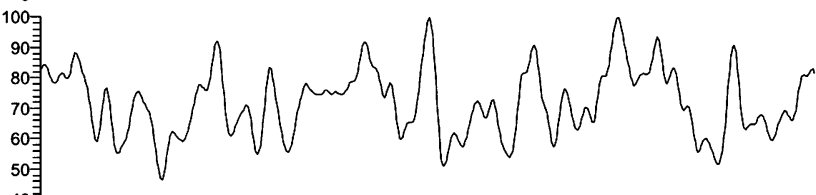

40 年

20年

10年

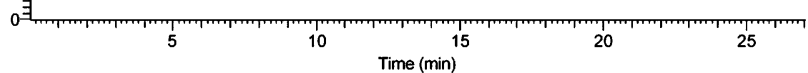

Figure 5. Evaluation of the crosstalk effect for the four-channel multiplex CE/MS system. Extracted ion electropherogram $(\mathrm{m} / \mathrm{z}=283)$ from four events. Sample: column 3, 2000 ppm niflumic acid ([M + $\mathrm{H}]^{+}=283$ ); the other three columns not injected. Conditions as described in Figure 3.

appears at the end of a complete scan. The signal (a 5-V pulsed signal) was sent to the analogue-to-digital converter of the motion control card and was used to control the operation of the stepper motor. At the end of a scan, the stepper motor was activated by the DRQ1 signal, and the open section of the metal disk was moved to the next sprayer before the start of the new scan. Occasionally, noise spikes were observed in the DRQ1 output. To withstand the effects from spikes, the threshold voltage was set to $3.5 \mathrm{~V}$, since the spike intensity rarely exceeded $2.5 \mathrm{~V}$. The time for the metal disk to rotate a $90^{\circ}$ was $<50 \mathrm{~ms}$, and the lag time between scans was found to be sufficient for the disk to rotate to the next position before acquiring a new scan.

In addition to the need for sheath liquid for each column, one major difficulty encountered in the design of a multiplexed CE/ MS interface is the limited space between the interface and the entrance aperture of the mass spectrometer. The flow rate of a sheath flow CE/MS $(3-5 \mu \mathrm{L} / \mathrm{min})$ is much smaller than that of multiplexed LC/MS (>200 $\mu \mathrm{L} / \mathrm{min}$ ). Therefore, to achieve good sensitivity, the space between column exit and entrance aperture is rather limited in multiplexed CE/MS interface. Consequently,

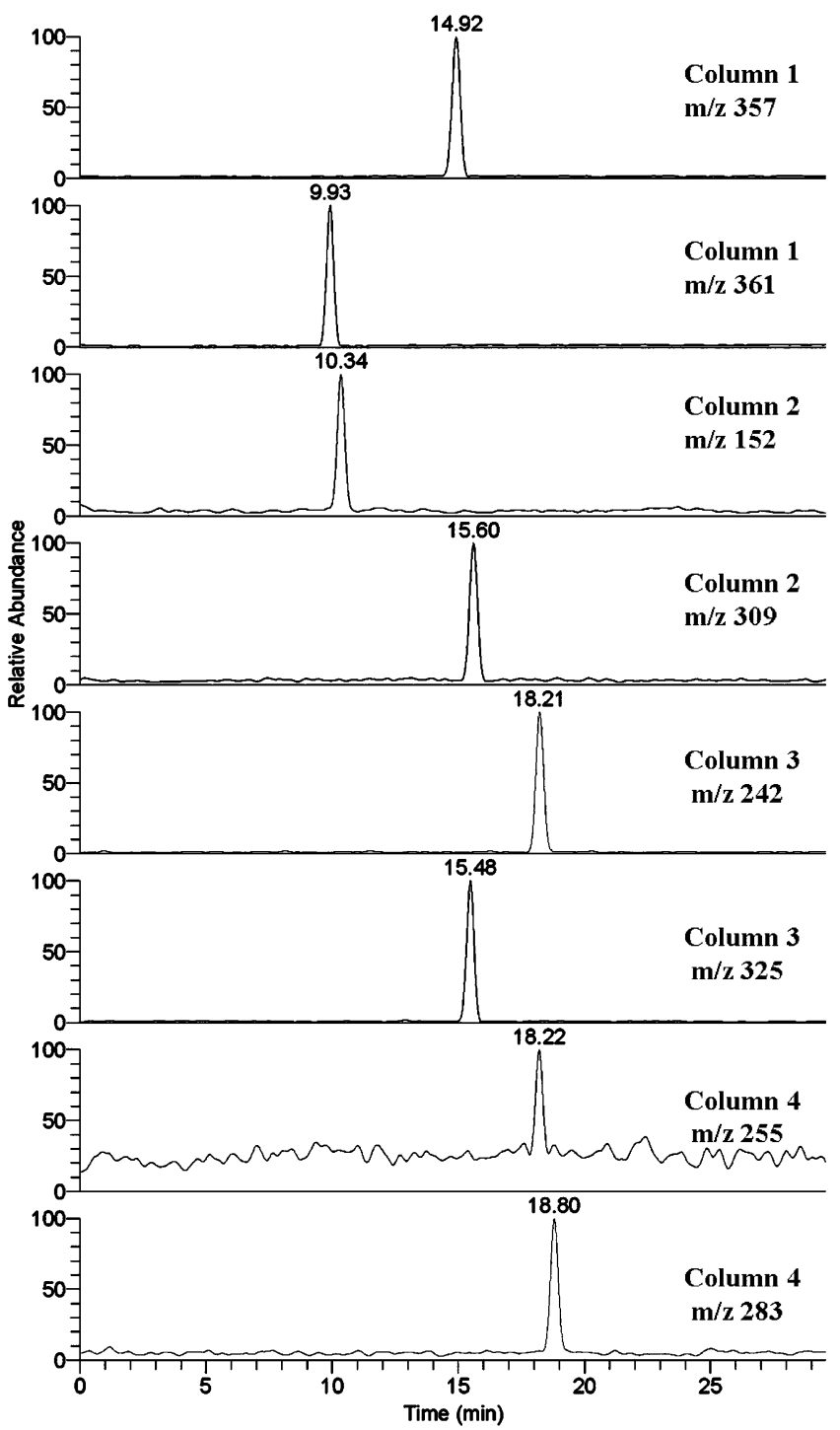

Figure 6. Multi-CE/ESI-MS analysis of four separation columns. Extracted ion electropherograms of the eight synthetic drugs. Sample: column 1, $250 \mathrm{ppm}$ sulindac $\left([\mathrm{M}+\mathrm{H}]^{+}=357\right)$ and prednisolone $\left([\mathrm{M}+\mathrm{H}]^{+}=361\right)$; column 2, $250 \mathrm{ppm}$ 4-acetaminophe$\mathrm{nol}\left([\mathrm{M}+\mathrm{H}]^{+}=152\right)$ and phenylbutazone $\left([\mathrm{M}+\mathrm{H}]^{+}=309\right)$; column $3,250 \mathrm{ppm}$ mefenamic acid $\left([\mathrm{M}+\mathrm{H}]^{+}=242\right)$ and oxyphenbutazone $\left([\mathrm{M}+\mathrm{H}]^{+}=325\right)$; column 4, 250 ppm ketoprofen $\left([\mathrm{M}+\mathrm{H}]^{+}=255\right)$ and niflumic acid $\left([\mathrm{M}+\mathrm{H}]^{+}=283\right)$. Conditions as described in Figure 5.

the evaluation of crosstalk between adjacent sprayers is essential in the evaluation of this multiplexed system. The selectivity of the multiplexed $\mathrm{CE} / \mathrm{MS}$ device was tested with the analysis of two synthetic drugs using two $\mathrm{CE}$ columns. Since the two sprayers were placed $180^{\circ}$ apart, the movement of the stepper motor was set to rotate $180^{\circ}$ after each scan. The mass electropherograms of the two $[\mathrm{M}+\mathrm{H}]^{+}$signals are shown in Figure 3. The observation of no $\mathrm{m} / z 361$ ion in sprayer 1 (Figure 3a) and no $m / z 357$ ion peak in sprayer 2 (Figure $3 \mathrm{~b}$ ) suggested that there was no crosstalk between the two sprayers.

In the four-sprayer configuration, however, some crosstalk was observed owing to the closer proximity of adjacent sprayers. In the current system, it was discovered that the relative position of the sprayers was critical to avoid interchannel crosstalk. A study of probe position versus crosstalk suggested that the arrangement 
shown in Figure $4 \mathrm{~b}$ was preferred over that shown in Figure $4 \mathrm{a}$ or $\mathrm{c}$. In configuration $4 \mathrm{a}$, although the sprayer was more favorably positioned relative to the mass spectrometer entrance aperture, crosstalk was often observed. It was discovered that as the sprayers were positioned closer to the rotating axle, the space between sprayers decreased, leading to higher crosstalk between neighboring sprayers. On the other hand, if the sprayers were arranged too far away from the rotating axle (Figure 4c), the sensitivity was found to be poor. To eliminate the problem of crosstalk and maintain good sampling efficiency, the configuration shown in Figure $4 \mathrm{~b}$ was found to be the most appropriate position. In configuration $4 \mathrm{~b}$, the sprayers were placed at the position where the tip of the sprayer is $\sim 5 \mathrm{~mm}$ from the disk and $10 \mathrm{~mm}$ from the axle. As indicated in the figure, the angle $(\theta)$ between the axle and the sprayer was $\sim 45^{\circ}$. Since the assembly (rotating disk) was $\sim 5 \mathrm{~mm}$ away from the mass spectrometer (entrance aperture), the distance between the sampling cone and the sprayer was $\sim 15 \mathrm{~mm}$. A distance greater than $15 \mathrm{~mm}$ was not recommended, because the intensity would decrease when the sprayer was positioned farther away from the mass spectrometer entrance aperture. To estimate the influence of crosstalk, a series of solutions with concentrations up to $2000 \mathrm{ppm}$ were studied. The results (Figure 5) showed that with the configuration $4 \mathrm{~b}$, the interchannel crosstalk was negligible, even at a concentration of $2000 \mathrm{ppm}$.

Another issue that needs to be addressed in a multiplexed system is cycle time. In general, $\sim 10$ data points are needed across a chromatographic peak without distortion. ${ }^{22}$ Therefore, the cycle time has to be short to enable 8 or 10 data points to be acquired across a chromatographic peak. In a multiplexed system, the cycle time depends mainly on the number of channels and the time for a regular scan. The time sequence of a regular scan has been studied using a digital oscilloscope. The trace showed that a default full scan comprises three microscans and that each microscan includes ion preinjection, prescan, ion injection, and mass scan. Following three microscans is a lag time which includes the time for uploading data to the data system and an interscan delay time. It was observed that the mass scan time and data-uploading time were fixed on the basis of the mass range of a scan. As mentioned earlier, the time for a complete scan in an ion trap is not a fixed value. The major source of the variation was found to be the ion injection time. In the LCQ instrument, a maximum injection time was selected before each $\mathrm{CE} / \mathrm{MS}$ analysis. It was observed that the actual injection time varied with concentration of the analyte. During the course of an electrophoretic peak, the ion injection time varied considerably because the concentration of the analyte changed greatly across an electrophoretic peak. This is also the reason why when an ion

(22) Ardrey, R. E. Liquid Chromatography-Mass Spectrometry: An Introduction; Wiley: Chichester, U.K., 2003. trap mass spectrometer is used as the detector, more data points are obtained on the top of a peak than on the shoulder of the peak.

When the default value for a regular scan (including three microscans) was used, the scan time and data uploading time for a 300-amu scan $(m / z 100-400)$ was $\sim 0.46$ s. If the maximum ion injection time was set to $100 \mathrm{~ms}$, the time for a complete scan should be somewhere between 0.46 and $0.76 \mathrm{~s}$, depending on the actual ion injection time. Therefore, the cycle time for a fourchannel system would be between 1.84 and $3.04 \mathrm{~s}$. To evaluate the cycle-time effect, a four-sprayer setup with eight synthetic drugs was tested. The resulting extracted ion electropherogram of the eight $[\mathrm{M}+\mathrm{H}]^{+}$signals are shown in Figure 6 . As can be seen, the peak integrity was preserved under the conditions employed. The 1.84-3.04-s cycle time may not be short enough if more than four sprayers are mounted on the assembly. There are two ways to reduce the cycle time: one is to reduce the number of microscans, and the other is to reduce the maximum ion injection time. A low value for the maximum ion injection time would reduce sensitivity, resulting in a failure to detect low-level analytes. Therefore, it would be more effective to change the cycle time by reducing the number of microscans. It was observed that when the number of microscans was reduced to two or one, the maximum scan time was reduced to 0.29 and $0.53 \mathrm{~s}$, respectively. Accordingly, the maximum cycle time was reduced to 1.16 and $2.12 \mathrm{~s}$, respectively. Under these conditions, at the expense of a lower $\mathrm{S} / \mathrm{N}$ ratio, a 20 -s peak would produce enough data points to maintain the peak integrity.

\section{CONCLUSIONS}

A multiplexed sheath-flow CE/MS interface coupled to an ion trap mass spectrometer has been developed. The capability of performing parallel separation with parallel mass spectrometric detection was evaluated. When appropriately configured, this system is capable of enhancing throughput 4-fold without interchannel crosstalk. One limitation of this design is that the maximum number of parallel $\mathrm{CE}$ columns is limited to about four. This restriction is primarily due to interchannel crosstalk, since there is not enough space to accommodate more than four sprayers. Another problem encountered when using a system with more than four sprayers is a data acquisition rate to ensure that chromatographic integrity is maintained. This limitation could be addressed, but it would require the use of a mass spectrometer that is capable of faster data acquisition rates.

\section{ACKNOWLEDGMENT}

This work was supported by the National Research Council of the Republic of China.

Received for review December 23, 2005. Accepted April 21, 2006.

AC052272W 